\title{
Prevalência de doença cardiovascular numa população de doentes com síndrome de apneia obstrutiva do sono
}

José Pedro Boléo-Tomé,* Sara Salgado, ${ }^{* *}$ Ana Sofia Oliveira, ${ }^{* * *}$ Paula Pinto, ${ }^{* * * *}$ António Bugalho, ${ }^{* * * * *}$ Amélia Feliciano, ${ }^{* * *}$ Cristina Canhão, ${ }^{* * * * * *}$ Ana Rita Tavares Dias, ${ }^{* * * * * *}$ Joana Isaac Teixeira, ${ }^{* * * * * *}$ Cristina Bárbara*******

\section{RESUMO}

Introdução: A Síndrome de Apneia Obstrutiva do Sono (SAOS) induz uma série de alterações fisiopatológicas que aumentam o risco de desenvolvimento de doenças cardiovasculares. Entre estas, a hipertensão arterial (HTA) tem sido a mais estudada e cuja associação causal está mais bem estabelecida. Existem poucos dados sobre a prevalência destas patologias nos doentes com SAOS em Portugal.

Tipo de estudo: Análise retrospectiva.

Métodos: Determinação da prevalência de patologias cardiovasculares e sua relação com os vários graus de gravidade da SAOS, mediante a análise dos processos de 305 doentes com diagnóstico de SAOS seguidos na Consulta de Patologia do Sono do nosso Hospital.

Resultados: Verificou-se uma prevalência de doenças cardiovasculares de $76,7 \%$, sendo a HTA a mais frequente (60\%), seguida da cardiopatia isquémica $(11,8 \%)$ e das disritmias $(10,8 \%)$. Outros factores de risco cardiovasculares como a obesidade $(61,3 \%)$, diabetes $(20,3 \%)$, dislipidemia $(43,3 \%)$ e tabagismo $(49,8 \%)$ foram também encontrados num elevado número de doentes.

Conclusão: Detectou-se uma elevada prevalência de HTA, pelo que num doente com o diagnóstico de HTA deve ser sempre avaliada a possibilidade de existência de SAOS. Por outro lado, a constatação de uma prevalência elevada de outros factores de risco cardiovascular potencia uma maior morbilidade e mortalidade nos doentes com SAOS.

Palavras-chave: Síndrome de Apneia Obstrutiva do Sono; Doença Cardiovascular; Hipertensão

*Interno do Internato Complementar de Pneumologia, Centro Hospitalar de Lisboa Ocidental, EPE - Hospital Egas Moniz

**Interna do Internato Complementar de Pneumologia, Hospital Pulido Valente ***Assistente Hospitalar de Pneumologia, Hospital Pulido Valente

****Assistente Hospitalar de Pneumologia, Hospital Pulido Valente; Assistente Convidada da Faculdade de Ciências Médicas de Lisboa; Investigadora do Centro de Estudos de Patologia Respiratória da Fundação para a Ciência e Tecnologia *****Assistente Hospitalar de Pneumologia, Hospital Pulido Valente; Assistente Convidado da Faculdade de Ciências Médicas de Lisboa; Investigador do Centro de Estudos de Patologia Respiratória da Fundação para a Ciência e a Tecnologia *******Técnica de Cardiopneumologia, Hospital Pulido Valente

*******Assistente Hospitalar Graduada de Pneumologia; Coordenadora da Unidade de Fisiopatologia Respiratória, Hospital Pulido Valente; Professora Auxiliar Convidada da Faculdade de Ciências Médicas de Lisboa; Investigadora do Centro de Estudos de Patologia Respiratória da Fundação para a Ciência e a Tecnologia

Instituição: Unidade de Fisiopatologia Respiratória - Departamento de Pneumologia Hospital Pulido Valente, Lisboa

\section{INTRODUÇÃO}

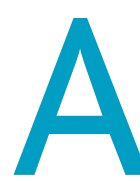

Síndrome de Apneia Obstrutiva do Sono (SAOS) é uma condição clínica frequente que se caracteriza por episódios repetidos de obstrução da via aérea superior durante o sono, associados a aumento do esforço respiratório, dessaturação de oxigénio intermitente e fragmentação do sono, com activação do sistema nervoso autónomo. ${ }^{1,2}$

Os efeitos cardiovasculares associados à SAOS têm vindo a ser descritos desde há longa data. A hipóxia nocturna repetida está associada a activação de uma série de mecanismos neuronais, humorais, inflamatórios e trombóticos, que têm sido implicados na patogénese das complicações cardiovasculares. ${ }^{3}$ 
A SAOS está associada à obesidade ${ }^{3,4}$ e está frequentemente presente em doentes com doença cardiovascular, nomeadamente a hipertensão arterial (HTA), ${ }^{3-7}$ disritmias, ${ }^{8,9}$ insuficiência cardíaca congestiva (ICC), ${ }^{10,11}$ cardiopatia isquémica ${ }^{3,12}$ e acidente vascular cerebral (AVC) ${ }^{13,14}$ A associação à HTA tem sido a mais estudada e a SAOS constitui um factor de risco independente para a sua génese, com ampla evidência publicada. . $^{1,45}$ A hiperactividade simpática desencadeada pela SAOS é, provavelmente, um dos mecanismos responsáveis pelo desenvolvimento de HTA, que persiste durante o dia $^{15}$.Verificou-se também que o tratamento eficaz com pressão positiva contínua (Continuous Positive Airway Pressure - CPAP) conduz a uma redução da pressão arterial diurna e nocturna. ${ }^{16} \mathrm{~A}$ associação destas duas patologias justificou a inclusão da SAOS como causa de HTA secundária, em recentes recomendações sobre a abordagem diagnóstica da HTA. ${ }^{17}$

A relação causal entre a SAOS e outras patologias cardiovasculares é mais difícil de estabelecer, devido a numerosos factores confundidores. No entanto, recentemente foi demonstrado que a terapêutica com CPAP em doentes com apneia do sono reduziu o risco de doença cardiovascular fatal e não fatal. ${ }^{18}$ Assim, a importância da SAOS como um factor de risco cardiovascular potencialmente tratável deve ser realçada.

As relações entre a SAOS e a constelação de factores que constitui a síndrome metabólica têm também vindo a ser demonstradas e são reveladoras de uma grande complexidade de alterações fisiopatológicas. ${ }^{19}$

Em Portugal existem poucos dados sobre a epidemiologia da SAOS e complicações cardiovasculares. Um estudo de Drummond apontava uma prevalência de HTA de $45,3 \%$, numa população de 155 doentes. ${ }^{20} \mathrm{Na}$ literatura internacional têm sido referidas prevalências até $50 \%$ e tem sido diagnosticada SAOS em até $30 \%$ dos doentes hipertensos. ${ }^{2}$

Neste estudo pretendeu-se avaliar a prevalência das principais patologias cardiovasculares numa população de SAOS e analisar a relação entre a prevalência e os vários graus de gravidade da síndrome.

\section{MATERIAL E MÉTODOS}

Realizou-se uma análise retrospectiva dos processos clínicos de 305 doentes seguidos na Consulta de Patologia do Sono do hospital, com o diagnóstico de SAOS, avaliados entre os meses de Dezembro de 2005 e Julho de 2006. Para o efeito foram seleccionados todos os doentes com SAOS confirmada, independentemente de terem sido enviados à Consulta para diagnóstico ou já estarem em seguimento. Excluíram-se os doentes sem diagnóstico confirmado. Foram estudadas as características demográficas, dados antropométricos, Índice de Apneia/Hipopneia (IAH), CT90 (percentagem de tempo de sono com saturação periférica de oxigénio inferior a 90\%), presença de doença cardiovascular e factores de risco cardiovascular.

O diagnóstico de SAOS foi obtido através da realização de estudo polissonográfico ou de estudo cardio-respiratório do sono, consoante a indicação clínica e as características dos doentes. O estudo do sono era realizado em todos os doentes com queixas de hipersonolência diurna e/ou roncopatia, associadas ou não a apneias testemunhadas, sensação de asfixia ou sono não reparador. Os estudos foram realizados no Laboratório do Sono do nosso Hospital. Foram considerados três estádios de gravidade de SAOS, conforme consenso actual::21 SAOS ligeiro, definido por IAH $\geq 5$ e $<15$ eventos respiratórios/hora, SAOS moderado, definido por IAH $\geq 15$ e $<30$ e SAOS grave, definido por IAH $\geq 30$.

Os doentes foram estratificados conforme o Índice de Massa Corporal (IMC) calculado a partir do peso e altura, nos seguintes grupos: peso normal para um IMC $<25 \mathrm{~kg} /$ $/ \mathrm{m}^{2}$, Pré-obesidade para IMC $\geq 25 \mathrm{e}<30$, Obesidade Classe I para IMC $\geq 30$ e $<35$, Classe II $\geq 35$ e $<40$ e Classe III $\geq 40$. $^{22}$

Foi avaliada a presença de diagnósticos prévios de hipertensão arterial (HTA), cardiopatia isquémica, com ou sem enfarte do miocárdio prévio, disritmias, insuficiência cardíaca congestiva (ICC) e acidente vascular cerebral (AVC) prévio, a partir dos dados da história clínica, exame físico e exames complementares disponíveis. Foi também pesquisada a presença de outros factores de risco cardiovascular como a dislipidemia, diabetes e tabagismo presente ou passado.

Os dados foram analisados sob a forma de estatística descritiva, calculando a distribuição de frequências, médias, desvios padrão, e as médias foram comparadas utilizando o teste $t$ de Student, definindo-se um valor de $p<0,05$ como estatisticamente significativo.

\section{RESULTADOS}

Foram estudados 305 doentes, dos quais $78 \%$ eram do 
sexo masculino, com uma idade média de 57,8 anos (Quadro I). O IMC variou entre 18 e $73 \mathrm{~kg} / \mathrm{m}^{2}$, com uma média de 32,3 (intervalo de confiança de 95\% - IC 95\%: 31,6 - 33,0). Quando divididos em classes, apenas 7,2\% dos doentes apresentavam um IMC normal (20-25 kg/ $/ \mathrm{m}^{2}$ ) e $61,3 \%$ apresentavam obesidade de grau variável (Figura 1); em 8,9\% dos casos, tratava-se de obesidade Classe III.

Todos os doentes apresentavam diagnóstico de SAOS de gravidade variável, com um IAH mínimo de 5 e máximo de 110 eventos respiratórios/hora. Conforme se constata na Figura 2, em mais de metade dos casos o IAH foi superior a 30 eventos por hora, correspondendo a SAOS grave.

O CT90 variou entre 0 e 100\%, sendo a média de 24,5\% (IC 95\%: 21,0-28,0). Quando comparadas as médias do CT90 nos três escalões de gravidade de SAOS, esta foi consideravelmente superior nos estádios mais graves $(35,5 \% \pm 32,7$ para um $\mathrm{IAH} \geq 30$, comparado com $9,5 \% \pm 20,4$ para um IAH $<15$ ).

Quanto às doenças associadas, verificou-se que 234 doentes $(76,7 \%)$ apresentavam algum tipo de patologia cardiovascular, conforme representa a Figura 3. Destas patologias, a mais prevalente foi a HTA com 183 doentes, correspondendo a $60 \%$ dos mesmos, seguida da cardiopatia isquémica ( 36 casos) e das disritmias (33 casos). Destas, a fibrilhação auricular (FA) foi a mais frequente, estando presente em 25 doentes. A ICC estava presente em 11 doentes e 8 já tinham tido um ou mais episódios de AVC.

\begin{tabular}{|c|c|c|c|}
\hline \multirow{2}{*}{\multicolumn{4}{|c|}{$\begin{array}{l}\text { QUADRO I. Descrição da população est } \\
\text { desvio padrão, valor mínimo e máximo } \\
\text { confiança de } 95 \% \text { (IC } 95 \% \text { ) } \\
\text { Características da população estudada }\end{array}$}} \\
\hline & & & \\
\hline & $\begin{array}{c}\text { Média } \pm \\
\text { Desvio padrão }\end{array}$ & $\begin{array}{l}\text { Mínimo/ } \\
\text { Máximo }\end{array}$ & IC 95\% \\
\hline $\begin{array}{l}\text { Idade média } \\
\text { (anos) }\end{array}$ & $57,8 \pm 10,7$ & 21 a 82 & $56,6-59,0$ \\
\hline $\begin{array}{l}\text { IMC médio } \\
\left(\mathrm{Kg} / \mathrm{m}^{2}\right)\end{array}$ & $32,3 \pm 6,6$ & 18 a 73 & $31,6-33,0$ \\
\hline $\begin{array}{l}\text { IAH médio } \\
\text { (eventos/hora) }\end{array}$ & $36,4 \pm 22,9$ & 5 a 110 & $33,8-39,0$ \\
\hline CT 90 médio (\%) & $24,5 \pm 31,1$ & 0 a 100 & $21,0-28,0$ \\
\hline
\end{tabular}

Na avaliação de outros factores de risco cardiovascular (Figura 4), verificou-se a existência de dislipidemia em $43,3 \%$ dos doentes e diabetes mellitus em $20,3 \%$. Uma história de tabagismo actual ou passado estava presente em $49,8 \%$ dos casos, mantendo ainda 22,3\% dos doentes os hábitos tabágicos.

Verificou-se que a percentagem de doentes hipertensos aumentava com a gravidade da SAOS: para um $\mathrm{IAH}<15$, encontrámos $52,5 \%$ de hipertensos, enquan-

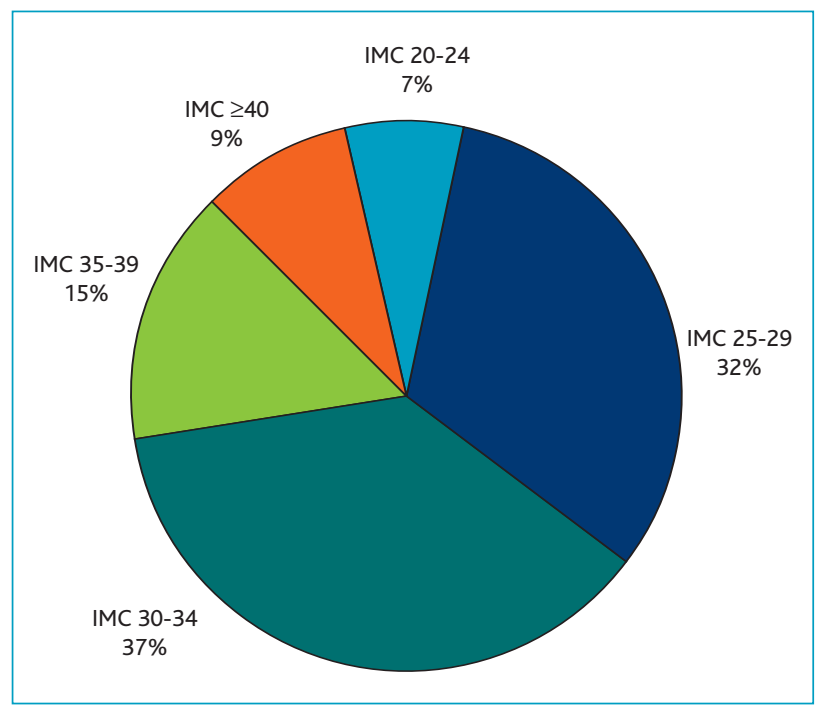

Figura 1. Classificação dos doentes por classes de obesidade IMC: Índice de Massa Corporal.

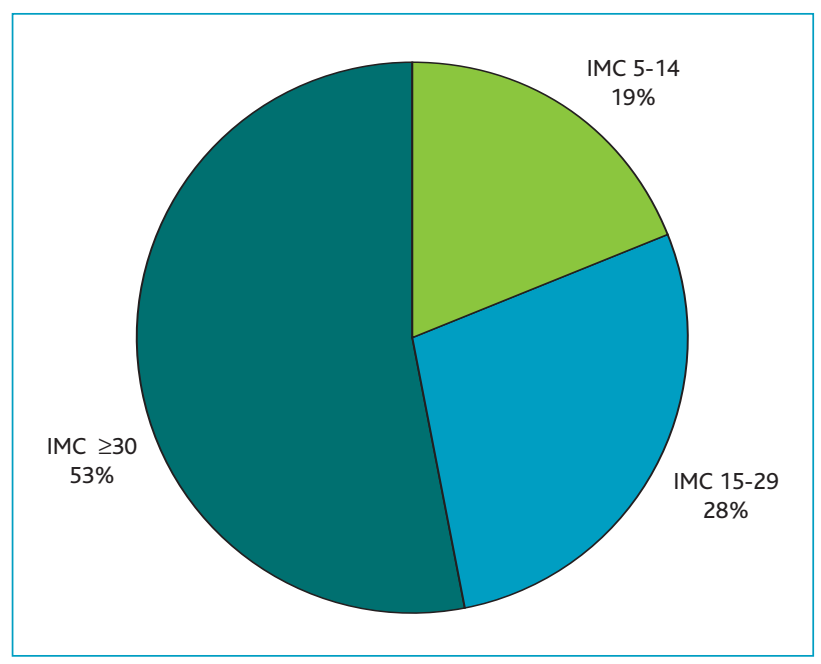

Figura 2. Classificação da população em estadios de gravidade da SAOS. IAH: Índice de Apneia/Hipopneia 


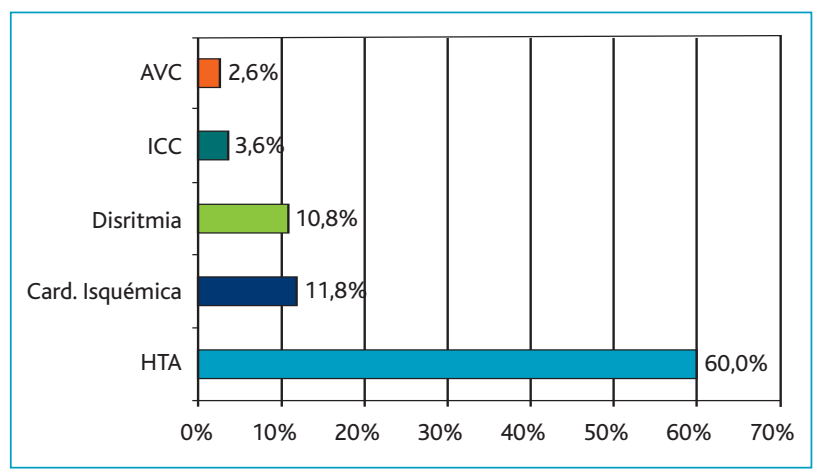

Figura 3. Prevalências de patologias cardiovasculares.

AVC: acidente vascular cerebral; ICC: insuficiência cardíaca congestiva; HTA: hipertensão arterial.

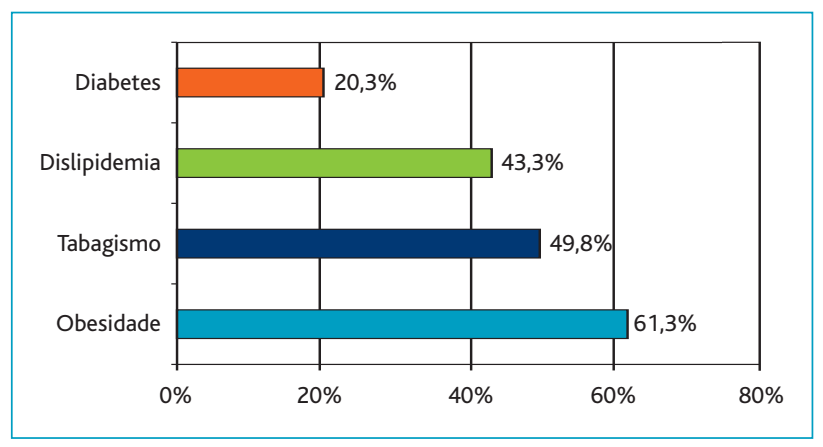

Figura 4. Prevalência de outros factores de risco cardiovascular.

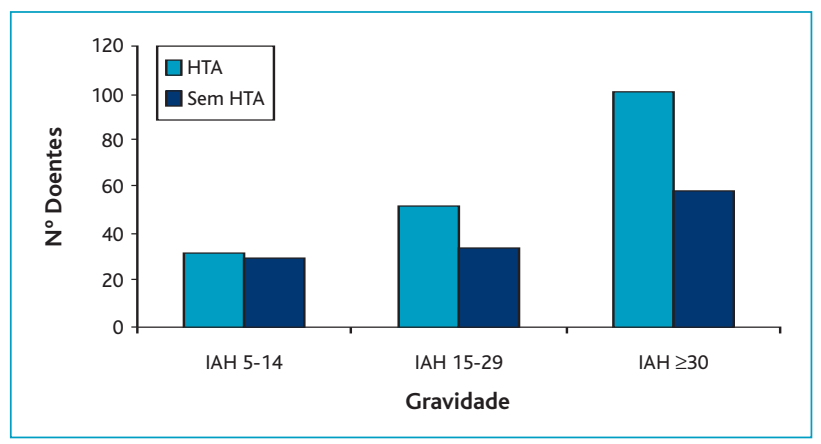

Figura 5. Relação entre prevalência de HTA e estadio de gravidade da SAOS.

to para um $\mathrm{IAH} \geq 30$ existiam $62,3 \%$ de hipertensos (Figura 5).

Foi analisada a média do IAH para cada um dos grupos de doentes com ou sem doença cardiovascular associada e não foram encontradas diferenças significativas. Apenas foi encontrado um IAH médio mais baixo no grupo dos doentes que já tinham tido AVC em re-

\begin{tabular}{|c|c|c|c|}
\hline \multicolumn{4}{|c|}{$\begin{array}{l}\text { QUADRO II. Análise comparativa entre as } \\
\text { médias de IAH nos indivíduos com e sem doença } \\
\text { cardiovascular, para cada uma das patologias avaliadas } \\
\text { (n.s.: não significativo) }\end{array}$} \\
\hline & \multicolumn{2}{|c|}{ Média IAH } & \multirow[b]{2}{*}{$p$} \\
\hline Patologia & Com doença & Sem doença & \\
\hline HTA & 35,4 & 37,9 & 0,349 (n.s.) \\
\hline $\begin{array}{l}\text { Cardiopatia } \\
\text { isquémica }\end{array}$ & 37,3 & 29,1 & 0,053 (n.s.) \\
\hline Disritmia & 30,6 & 37,0 & 0,148 (n.s.) \\
\hline ICC & 27,1 & 36,6 & 0,245 (n.s.) \\
\hline AVC & 14,0 & 36,8 & 0,027 \\
\hline Diabetes & 35,0 & 36,7 & 0,609 (n.s.) \\
\hline Dislipidemia & 34,6 & 37,7 & 0,239 (n.s.) \\
\hline Tabagismo & 34,5 & 38,3 & 0,152 (n.s.) \\
\hline
\end{tabular}

lação aos que nunca tinham tido $(14,0$ vs. 36,$8 ; \mathrm{p}=$ 0,027). A análise comparativa das diferentes médias encontra-se representada no Quadro II.

\section{DISCUSSÃO E CONCLUSÕES}

Os dados recolhidos neste estudo apontam para uma elevada prevalência de doença cardiovascular neste grupo de doentes. A patologia mais frequente foi sem dúvida a HTA, com uma prevalência global de $60 \%$. Foi já estabelecido que um IAH igual ou superior a 15 está associado a um risco três vezes maior de desenvolvimento de HTA. ${ }^{3,5}$ No grupo estudado, a prevalência de HTA aumentou com a gravidade da SAOS, mas mesmo nos estádios ligeiros (IAH 5 a 15) a prevalência encontrada foi importante (52,5\%). Estes dados podem explicar-se pela relação dose-dependente entre apneia do sono e desenvolvimento de HTA, conforme foi demonstrado pelo Estudo Wisconsin e outros mais recentes. ${ }^{5-7,12}$

A população estudada apresenta uma gravidade de SAOS significativa, com uma elevada percentagem de casos pertencentes ao estádio grave (53\%). Como seria de esperar, os estádios de maior gravidade correspondem a valores superiores de CT90, embora haja uma grande dispersão de valores: alguns doentes graves não dessaturavam e outros menos graves apresentavam dessaturações consideráveis.

Provavelmente haverá uma percentagem importan- 
te de casos considerados como HTA essencial que corresponderão a HTA secundária a SAOS não diagnosticada ou tratada. ${ }^{3}$

No follow-up dos doentes hipertensos após início da terapêutica com CPAP verifica-se frequentemente uma normalização dos valores tensionais diurnos ou redução do número e da dose de anti-hipertensores necessários. ${ }^{16,23} \mathrm{~A}$ introdução de CPAP produz inclusivamente um efeito de «primeira noite» em que se assiste a uma redução imediata da pressão arterial. ${ }^{24}$

As prevalências encontradas para as outras patologias estudadas são bastante inferiores à HTA e tem sido mais difícil de estabelecer uma relação causal com a SAOS.

A cardiopatia isquémica surge em $11,8 \%$ dos casos e poderá estar em parte relacionada com a cascata de alterações inflamatórias e procoagulantes, associadas à hipóxia e hipercápnia nocturnas. O Sleep Heart Health Study também já incluiu a SAOS como um factor de risco independente para doença coronária. ${ }^{12} \mathrm{~A}$ presença de SAOS pode também ser um indicador prognóstico nos doentes que tiveram enfarte do miocárdio, associando-se a uma maior mortalidade. ${ }^{25}$ Neste estudo, apesar da diferença não ser estatisticamente significativa, verificou-se uma tendência para um IAH médio superior nos doentes com cardiopatia isquémica, em comparação com os restantes doentes $(37,3$ vs. 29,1).

As disritmias cardíacas têm sido descritas frequentemente em doentes com SAOS, nomeadamente os bloqueios auriculo-ventriculares, FA e extrassistolia ventricular; no entanto, as bradiarritmias são as que parecem estar mais directamente relacionadas com a apneia e a hipóxia. ${ }^{26}$ Grande parte dos casos encontrados neste grupo correspondem a FA, o que poderá ser apenas um achado ou traduzir uma amostra demasiado reduzida para ser representativa. Há, no entanto, estudos que referem uma associação significativa entre SAOS e FA, encontrando-se uma prevalência de FA mais elevada em doentes com SAOS do que em doentes com outras patologias cardiovasculares. ${ }^{8}$

$\mathrm{O}$ facto de se ter verificado que os doentes com AVC prévio apresentavam um IAH inferior ao dos doentes sem antecedentes prévios de AVC poderá decorrer do número reduzido de doentes (oito) e de os doentes com sequelas graves de AVC não estarem incluídos nesta amostra, por nem sequer recorrerem a uma consulta deste tipo. De qualquer forma, os doentes com SAOS têm um risco mais elevado de terem AVC. ${ }^{13}$ Por outro lado, a apneia do sono tem uma prevalência elevada nos doentes com doença cérebro-vascular. ${ }^{27}$

É também de destacar a elevada prevalência de outros factores associados a risco cardiovascular: a dislipidemia em $43,3 \%$ dos casos e o tabagismo em $49,8 \%$ dos casos. Também a análise do IMC traduz uma população marcadamente obesa, como é habitual encontrar nesta patologia. Além da forte relação causal da obesidade com a apneia do sono, tem surgido evidência de que a própria SAOS pode predispor ao aumento de peso, mecanismo no qual a leptina pode desempenhar um papel importante. ${ }^{28}$

A coexistência destes factores com a SAOS aumenta por si só significativamente o risco de desenvolvimento de outras doenças cardiovasculares e o risco de eventos fatais relacionados com doença cardiovascular. A associação destes múltiplos factores em muitos doentes com SAOS torna difícil a sua análise e a identificação de factores de risco independentes.

Recentemente tem sido constatada a associação entre a SAOS e a síndrome metabólica ou síndrome X. ${ }^{19}$ Esta é uma entidade clínica definida pela presença de pelo menos três dos seguintes critérios: 1) obesidade abdominal (aumento do perímetro abdominal); 2) triglicéridos elevados (>150 mg/dL);3) diminuição do colesterol HDL ( $<40 \mathrm{mg} / \mathrm{dL}$ no homem ou $<50 \mathrm{mg} / \mathrm{dL}$ na mulher); 4) HTA; 5) glicemia em jejum superior a 110 $\mathrm{mg} / \mathrm{dL} .{ }^{29}$

A relação causal entre a SAOS e alguns componentes da síndrome metabólica, como é o caso da HTA, está bem estabelecida. A obesidade é um factor de risco reconhecido para a SAOS, mas tem sido também levantada a hipótese desta associação ser recíproca e a SAOS contribuir para o aumento de gordura e sobretudo para a redistribuição da adiposidade abdominal..$^{19}$

Neste estudo não foi analisado o perfil lipídico de cada doente, dispondo-se apenas de dados retrospectivos sobre a existência de dislipidemia. No entanto, parece existir uma relação inversa entre o IAH e os níveis de colesterol HDL e, ao mesmo tempo, uma relação directa com os níveis de triglicéridos. ${ }^{19}$

Finalmente, há alguma evidência que aponta para uma relação directa entre a SAOS e a resistência à insulina, independentemente da obesidade. Outras ligações fisiopatológicas entre a SAOS e a síndrome meta- 
bólica envolvem a inflamação sistémica, stress oxidativo, disfunção endotelial e hipercoagulabilidade. ${ }^{19}$ No entanto, é de notar que a maior parte destes dados provêm de estudos observacionais, não controlados ou com amostras pequenas.

Em conclusão, nesta população encontrámos uma elevada prevalência de doença cardiovascular, destacando-se a HTA que inclusive apresenta um valor superior à de algumas séries publicadas. As características deste trabalho não permitem estabelecer um nexo de causalidade entre a SAOS e as patologias cardiovasculares que lhe estão associadas, sendo necessários estudos prospectivos de longa duração alargados, nomeadamente na população portuguesa, para obter dados mais precisos. Contudo, a evidência científica aponta para a existência de uma relação causal entre SAOS e HTA, que motivou a recente modificação nas recomendações da abordagem diagnóstica da HTA. ${ }^{17}$ A constatação de uma prevalência elevada de outros factores de risco cardiovascular potencia uma maior morbilidade e mortalidade nos doentes com SAOS.

Tendo em conta estes resultados e os da literatura internacional, deve ser sempre avaliada a possibilidade de existência de SAOS num doente com o diagnóstico de HTA. Os doentes hipertensos com clínica sugestiva de SAOS, bem como os doentes com HTA refractária à terapêutica, deverão ser enviados a um centro especializado em patologia do sono para realização de exame diagnóstico. Nas restantes patologias cardiovasculares, também deve ser investigada a presença de sintomas sugestivos de SAOS. Assim, face às elevadas prevalências das patologias consideradas, a sua correcta abordagem implicará seguramente uma reestruturação do sistema de saúde de modo a facilitar a acessibilidade destes doentes a centros de sono.

\section{REFERÊNCIAS}

1. McNicholas WT, Bonsignore MR; Management Commitee of EU COST ACTION B26. Sleep apnoea as an independent risk factor for cardiovascular disease: current evidence, basic mechanisms and research priorities. Eur Respir J 2007 Jan; 29 (1): 156-78.

2. Thurnheer R. Obstructive sleep apnea and cardiovascular disease - time to act! Swiss Med Wkly 2007 Apr 21; 137 (15-16): 217-22.

3. Shamsuzzaman AS, Gersh BJ, Somers VK. Obstrutive sleep apnea: implications for cardiac and vascular disease. JAMA 2003 Oct 8;290(14):1906-14.

4. Wolk R, Shamsuzzaman AS, Somers VK. Obesity, sleep apnea, and hypertension. Hypertension 2003 Dec; 42 (6): 1067-74.

5. Peppard PE, Young T, Palta M, Skatrud J. Prospective study of the asso- ciation between sleep-disordered breathing and hypertension. N Engl J Med 2000 may 11; 342 (19): 1378-84.

6. Robinson GV, Stradling JR, Davies RJ. Obstructive sleep apnoea/hypopnoea syndrome and hypertension. Thorax 2004 Dec; 59 (12): 1089-94.

7. Dart RA, Gregoire JR, Gutterman DD, Woolf SH. The association of hypertension and secondary cardiovascular disease with sleep-disordered breathing. Chest 2003 Jan; 123 (1): 244-60.

8. Gami AP, Pressman G, Caples SM, Kanagala R, Gard JJ, Davison DE, et al. Association of atrial fibrillation and obstructive sleep apnea. Circulation 2004 Jul 27; 110 84): 364-7.

9. Kanagala R, Murali NS, Friedman PA, Ammash NM, Gersh BJ, Ballman $\mathrm{KV}$, et al. Obstructive sleep apnea and the recurrence of atrial fibrillation. Circulation 2003 May 27; 107 (20): 2589-94.

10. Schulz R, Blau A, Börgel J, Duchna HW, Fietze I, Koper I, et al. Sleep apnea in heart failure. Eur Respir J 2007 Jun; 29 (6): 1201-5.

11. Laaban JP, Pascal-Sebaoun S, Bloch E, Orvoën-Frija E, Oppert JM, Huchon $\mathrm{G}$. Left ventricular systolic dysfunction in patients with obstructive sleep apnea syndrome. Chest 2002 Oct; 122 (4): 1133-8.

12. Shahar E, Whitney CW, Redline S, Lee ET, Newman AB, Javier Nieto F, et al. Sleep-disordered breathing and cardiovascular disease: cross-sectional results of the Sleep Heart Health Study. Am J Respir Crit Care Med 2001 Jan; 163 (1):19-25.

13. Yaggi HK, Concato J, Kernan WN, Lichtman JH, Brass LM, Mohsenin V. Obstructive sleep apnea as a risk factor for stroke and death. N Engl J Med 2005 Nov 10; 353 (19): 2034-41.

14. Partinen M. Ischaemic stroke, snoring and obstructive sleep apnea. J Sleep Res 1995 Jun; 4 (S1):156-9.

15. Wieber $\mathrm{S}$. The cardiac consequences of the obstructive sleep apneahypopnea syndrome. Mt Sinai J Med 2005 Jan; 72 (1):10-2.

16. Becker HF, Jerrentrup A, Ploch T, Grote L, Penzel T, Sullivan CE, et al. Effect of nasal continuous positive airway pressure treatment on blood pressure in patients with obstructive sleep apnea. Circulation $2003 \mathrm{Jan}$ 7; 107 (1): 68-73.

17. 2007 Guidelines for the management of arterial hypertension. The task force for the management of arterial hypertension of the European Society of Hypertension (ESH) and of the European Society of Cardiology (ESC). J Hypertension 2007 Jun; 25 (6): 1105-1187.

18. Marin JM, Carrizo SJ, Vicente E,Agusti AG. Long-term cardiovascular outcomes in men with obstructive sleep apnoea-hypopnoea with or without treatment with continuous positive airway pressure: an observational study. Lancet 2005 Mar 19-25; 365 (9464): 1046-53.

19. Wolk R, Somers K. Sleep and the metabolic syndrome. Exp Physiol 2007 Jan; 92 (1):67-78.

20. Drummond M, Winck JC, Pereira S, Almeida J, Marques JA. Síndroma de apneia obstrutiva do sono e doença cardiovascular: estudo retrospectivo. Rev Port Pneumol 2003; 9 (4): 327-35.

21. Young T, Peppard PE, Gottlieb DJ. Epidemiology of obstructive sleep apnea: a population health perspective. Am J Respir Crit Care Med 2002 May 1; 165 (9):1217-39.

22. Clinical guidelines on the identification, evaluation and treatment of overweight and obesity in adults. Bethesda, Maryland: National Institutes of Health, National Heart, Lung and Blood Institute; 1998.

23. Hla KM, Skatrud JB, Finn L, Palta M, Young T. The effect of correction of sleep-disordered breathing on BP in untreated hypertension. Chest 
2002 Oct; 122 (4): 1125-32.

24. Pepperell JC, Ramdassingh-Dow S, Crosthwaite N, Mullins R, Jenkinson C, Stradling JR, et al. Ambulatory blood pressure after therapeutic and subtherapeutic nasal continuous positive airway pressure for obstructive sleep apnoea: a randomised parallel trial. Lancet 2002 Jan 19; 359 (9302): 204-10.

25. Peker Y, Hedner J, Kraiczi H, Löth S. Respiratory disturbance index: an independent predictor of mortality in coronary heart disease. Am J Respir Crit Care Med 2000 Jul; 162 (1): 81-6.

26. Zwillich C, Devlin T, White D, Douglas N, Weil J, Martin R. Bradycardia during sleep apnea: characteristics and mechanism. J Clin Invest 1982 Jun; 69 (6): 1286-92.

27. Wessendorf TE, Dahm C, Teschler H. Prevalence and clinical importance of sleep apnea in the first night after cerebral infarction. Neurology 2003 Mar; 60 (6): 1053.

28. Phillips BG, Kato M, Narkiewicz K, Choe I, Somers VK. Increases in leptin levels, sympathetic drive, and weight gain in obstructive sleep ap- nea. Am J Physiol 2000 Jul; 279 (1): H234-7.

29. Expert Panel on Detection, Evaluation, and Treatment of High Blood Cholesterol in Adults. Executive Summary of the Third Report of the National Cholesterol Evaluation Program (NCEP) Expert Panel on Detection, Evaluation and Treatment of High Blood Cholesterol in Adults (Adult Treatment Panel III). JAMA 2001 May 16; 285 (19): 2486-97.

\author{
ENDEREÇO PARA CORRESPONDÊNCIA \\ José Pedro Boléo-Tomé \\ Serviço de Pneumologia \\ Centro Hospitalar de Lisboa Ocidental, EPE - Hospital Egas Moniz \\ Rua da Junqueira, 126 - 1149-019 Lisboa \\ Tel: 967988700 \\ E-mail: jpboleotome@gmail.com
}

Recebido em 18/01/2008

Aceite para publicação em 27/06/2008

\section{ABSTRACT}

Introduction: Obstructive Sleep Apnea Syndrome (OSAS) leads to several pathophysiologic changes that increase the risk of developing cardiovascular disease. Among these, arterial hypertension has been the most studied and its causal association is well established. There are few data about the prevalence of these disorders in OSAS's patients in Portugal.

Type of study: Retrospective analysis.

Method: Determination of cardiovascular disease prevalence and its relationship with OSAS severity grades, through clinical record analysis of 305 patients diagnosed with OSAS and followed at a Sleep Outpatient Clinic.

Results: We found a prevalence of cardiovascular disease of $76,7 \%$, with hypertension being the most frequent (60\%), followed by ischaemic heart disease $(11,8 \%)$ and dysrythmias $(10,8 \%)$. Other cardiovascular risk factors like obesity $(61,3 \%)$, diabetes $(20,3 \%)$, dyslipidemia $(43,3 \%)$ and history of smoking $(49,8 \%)$ were also found in a high number of patients.

Conclusions: Given the high prevalence of hypertension found, every patient with a diagnosis of hypertension should be investigated for the possible presence of OSAS. Also the high frequency of other cardiovascular risk factors may lead to higher morbidity and mortality in OSAS patients.

Keywords: Obstructive Sleep Apnea Syndrome; Cardiovascular Disease; Hypertension. 